\title{
छூ
}

\section{Coherent Control of the Route of an Ultrafast Magnetic Phase Transition via Low-Amplitude Spin Precession}

\author{
J. A. de Jong, ${ }_{1}^{1}$ I. Razdolski, ${ }_{1}^{1}$ A. M. Kalashnikova, ${ }^{2}$ R. V. Pisarev, ${ }^{2}$ A. M. Balbashov, ${ }^{3}$ A. Kirilyuk, ${ }^{1}$ \\ Th. Rasing, ${ }^{1}$ and A. V. Kimel ${ }^{1}$ \\ ${ }^{1}$ Radboud University Nijmegen, Institute for Molecules and Materials, 6525 AJ Nijmegen, The Netherlands \\ ${ }^{2}$ Ioffe Physical-Technical Institute, Russian Academy of Sciences, 194021 St. Petersburg, Russia \\ ${ }^{3}$ Moscow Power Engineering Institute, 111250 Moscow, Russia
}

(Received 20 December 2011; published 9 April 2012)

\begin{abstract}
Time-resolved magneto-optical imaging of laser-excited rare-earth orthoferrite $(\mathrm{SmPr}) \mathrm{FeO}_{3}$ demonstrates that a single $60 \mathrm{fs}$ circularly polarized laser pulse is capable of creating a magnetic domain on a picosecond time scale with a magnetization direction determined by the helicity of light. Depending on the light intensity and sample temperature, pulses of the same helicity can create domains with opposite magnetizations. We argue that this phenomenon relies on a twofold effect of light which (i) instantaneously excites coherent low-amplitude spin precession and (ii) triggers a spin reorientation phase transition. The former dynamically breaks the equivalence between two otherwise degenerate states with opposite magnetizations in the high-temperature phase and thus controls the route of the phase transition.
\end{abstract}

PACS numbers: 77.80.B-, 75.50.Ee, 78.47. $-\mathrm{p}, 75.85 .+\mathrm{t}$

The kinetics of transitions between thermodynamically stable phases of matter is a subject of intense interest in a broad spectrum of research fields ranging from solid state physics to chemistry [1-3]. Conventionally, such transitions are triggered by a change of ambient conditions, such as temperature, pressure, and magnetic or electric fields. Controlling a phase of matter by an ultrashort (100 fs or less) optical excitation is both a new, rapidly developing research area and a counterintuitive phenomenon in which subtle excitations of atoms or spins are able to lead to dramatic changes in crystallographic, electric, or magnetic properties of media [4-6].

Furthermore, the feasibility of ultrafast control of transitions to a state described by a directional order parameter, such as magnetization or electric polarization, may have tremendous consequences for future recording and information processing, pushing these technologies into the range of unprecedented high frequencies above $10 \mathrm{GHz}$. One of the most straightforward approaches to achieve an ultrafast phase transition is to trigger it with the heating effect of a femtosecond laser pulse. This has been successfully employed for laser-induced spin reorientation in a broad class of materials including dielectrics [7], semiconductors [8], and metallic alloys $[9,10]$. It was shown, for instance, that excitation of a rare-earth (RE) orthoferrite with a 100 fs laser pulse may effectively change its magnetic anisotropy and trigger a subsequent $90^{\circ}$ spin reorientation within $10 \mathrm{ps}$ [7]. This opens a route for sub-100 ps spin manipulation in magnetic media.

That approach, however, has a serious drawback, since the high-temperature phase in these materials has at least two degenerate states, which are characterized by opposite orientations of the magnetic order parameter (i.e., magnetization "up" and "down," respectively). As a result, such a laser-induced phase transition from the low-temperature to the high-temperature phase proceeds along one of two equally probable routes, with the material eventually ending up in a multidomain state. In order to lift the degeneracy and thus to control the route of the transition, a magnetic field has to be present at the moment when the medium reaches the high-temperature phase [7-10].

In this Letter, we demonstrate a scenario for controlling the route of an ultrafast phase transition without any magnetic field present during the transition. Instead, the pure optical control is mediated by a coherent, long-living spin precession excited by the femtosecond laser pulse. In a striking difference with previously demonstrated mechanisms to control magnetic phase transitions, in this case laser pulses of the same helicity can create domains with opposite magnetization directions depending on the laser pulse fluence and the initial sample temperature.

For this study we have chosen single-crystalline $\left(\mathrm{Sm}_{0.5} \mathrm{Pr}_{0.5}\right) \mathrm{FeO}_{3}$ orthoferrite, grown by the floating-zone technique [11]. Owing to the balance between Sm and Pr contents, this orthoferrite possesses a low linear crystallographic birefringence, which results in a huge effective Faraday rotation [12,13]. In general, the iron $\mathrm{Fe}^{3+}$ ions in RE orthoferrites form two pairs of sublattices $S_{1}$ and $S_{2}$, which are coupled antiferromagnetically. Because of the Dzyaloshinsky-Moriya interaction, the spins are slightly canted, resulting in a weak magnetic moment [14,15]. Just below the Néel temperature $(\sim 700 \mathrm{~K})$, the spins are aligned along the $x$ axis with the weak magnetic moment along the $z$ axis. However, a number of orthoferrites, including $\mathrm{SmFeO}_{3}$, show a phase transition to a 
low-temperature phase in which the spins are aligned along the $z$ axis and the weak magnetic moment along the $x$ axis [see Fig. 1(a)]. We expect that such a phase transition also exists in $(\mathrm{SmPr}) \mathrm{FeO}_{3}$.

The sample studied here is a $z$-cut $93 \mu \mathrm{m}$-thick plate of $(\mathrm{SmPr}) \mathrm{FeO}_{3}$. For characterization of the magnetic properties of the sample, we measured the Faraday rotation by using a laser beam at the wavelength $\lambda=633 \mathrm{~nm}$, propagating along the $z$ axis. Since the birefringence is small, the rotation is proportional to the scalar product of the wave vector of light $\boldsymbol{k}$ and the magnetization of the sample $\boldsymbol{M}: \alpha_{F} \propto \boldsymbol{k} \cdot \boldsymbol{M}$. Figure 1(b) shows measurements of the static Faraday rotation in this sample and summarizes the behavior of the $z$ component of the magnetization $M_{z}$ at different temperatures. Above $T_{2} \approx 130 \mathrm{~K}$, the sample is in the high-temperature phase in which the magnetic moment is directed along the $z$ axis. The low-temperature phase, with the magnetic moment along the $x$ axis, is found below $T_{1} \approx 98 \mathrm{~K}$. In the phase transition region between $T_{1}$ and $T_{2}$, the magnetic moment rotates gradually in the $x z$ plane. The points $T_{1}$ and $T_{2}$ each mark a second-order phase transition.

In order to study the magnetization dynamics during the laser-induced phase transition, we used the technique of single-shot time-resolved magneto-optical imaging, similar to the one described elsewhere [16]. In short, the sample was excited with a 60 fs circularly polarized laser pulse at the wavelength of $800 \mathrm{~nm}$. This pump pulse was focused to a spot of $75 \mu \mathrm{m}$ full width at half maximum. We used pulses with energies in the microjoule range. Since the absorption in RE orthoferrites is relatively low in the nearinfrared [17], we estimate the laser-induced heating to

(a)
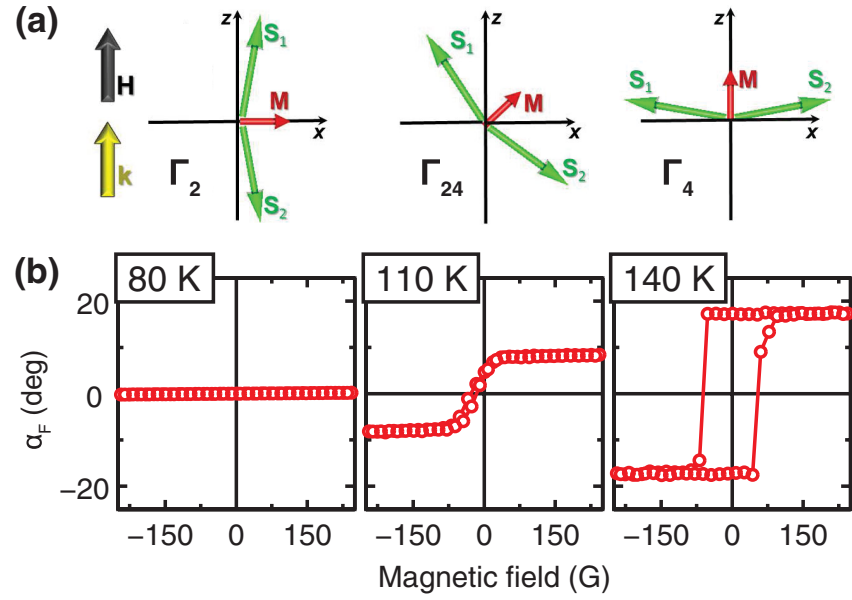

FIG. 1 (color online). (a) Magnetic structure of RE orthoferrites in the low-temperature $\left(\Gamma_{2}\right)$, intermediate $\left(\Gamma_{24}\right)$, and hightemperature $\left(\Gamma_{4}\right)$ phases. (b) Hysteresis of the $z$ component of the weak magnetization in $(\mathrm{SmPr}) \mathrm{FeO}_{3}$, probed by means of the Faraday rotation. The measurements show the presence of a phase transition region in which the net magnetic moment gradually rotates by $90^{\circ}$ from the $x$ axis to the $z$ axis. be only a few tens of degrees Kelvin in this area. The pump-induced changes in the material were probed by means of the Faraday rotation of a single, slightly longer, linearly polarized pulse at $633 \mathrm{~nm}$ and then recorded with a CCD camera. The measurements are sensitive to the outof-plane component of the magnetization $M_{z}$, such that black areas on the obtained magneto-optical images correspond to the domain with $M_{z}$ pointing down and white areas to the one with $M_{z}$ pointing up.

Figure 2 shows single-shot magneto-optical images obtained at different delays between the pump and probe pulses. The measurements were done for the sample temperature of $90 \mathrm{~K}$, just below the spin reorientation phase transition. At negative time delays, the images are gray, meaning that there is no $M_{z}$ component of the magnetization, as expected from the data shown in Fig. 1. An excitation of the sample with a left-handed circularly polarized pulse $\left(\sigma_{-}\right)$results in the formation of a "black" domain with $M_{z}$ pointing down within a few picoseconds. On the other hand, an excitation with a right-handed circularly polarized pulse $\left(\sigma_{+}\right)$creates a "white" domain with $M_{z}$ pointing up. We also used a linearly polarized pulse $(\pi)$ to excite the material and found that it leads to the formation of a multidomain state with relatively large black and white domains. The dark spot that is visible at $t=0$ is due to a short-lived pump-induced change in the transmittance of the sample. The measurements clearly show that a 60 fs circularly polarized laser pulse creates a homogeneous magnetic domain in the high-temperature

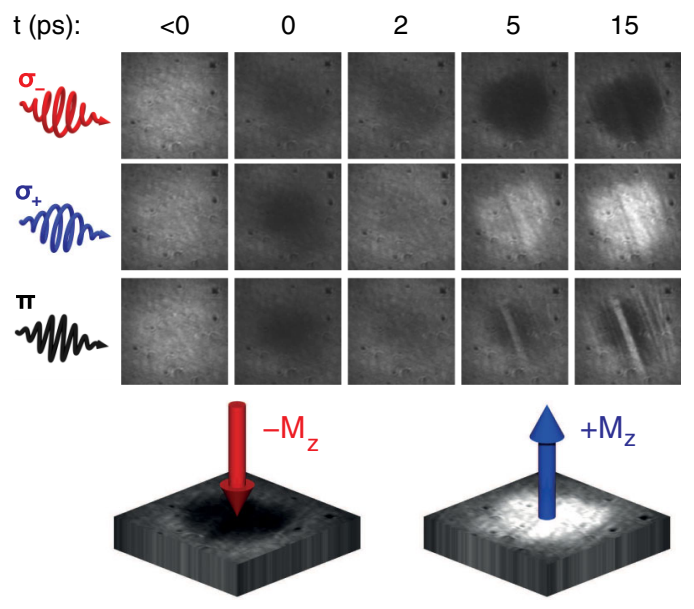

FIG. 2 (color online). Appearance of an out-of-plane component of the magnetization at a time scale of a few picoseconds due to excitation by a 60 fs laser pulse with energy $10.3 \mu \mathrm{J}$. In this case, the initial temperature of the sample was $90 \mathrm{~K}$. The out-of-plane component can point down (black) or up (white). Note that laser pulses with opposite helicities of the polarization create magnetic domains with opposite orientation of the magnetization. Linearly polarized laser pulses produce a multidomain state. Each picture shows an area of approximately $70 \times 70 \mu \mathrm{m}^{2}$. 
phase and that the magnetization direction in this domain is determined by the helicity of light.

Performing single-shot imaging of the laser-induced spin reorientation at different initial temperatures of the sample, we found that control over the direction of magnetization in the created domain is possible only if the sample temperature is below the temperature of the spin reorientation phase transition $T_{1}$. At higher temperatures within the spin reorientation transition interval, laser excitation can change only the absolute value of $M_{z}$ but not its sign. Above $T_{2}$, no effect of the femtosecond laser pulse is seen. We found that the created domains are present in the medium longer than $3 \mathrm{~ns}$ and usually disappear within $1 \mathrm{~ms}$. Note that the lifetime of the domain lies in the time window where the laser-heated area is expected to cool down to the initial temperature. From these observations, we conclude that the observed all-optical formation of the magnetic domains relies on laser-induced heating of the material over the point of the spin reorientation phase transition $T_{1}$ and the route of the transition can be controlled by the helicity of the femtosecond laser pulse.

The ultrafast heating of a RE orthoferrite with a femtosecond laser pulse can change the magnetic anisotropy on a time scale of 1-10 ps [18]. Such a change pushes the magnetization into one of two degenerate states with $M_{z}$ pointing up or down, respectively. However, even if the temperature of the sample is just a few degrees Kelvin below $T_{1}$, the $60 \mathrm{fs}$ pump pulse will have left the sample, and processes of optical decoherence will have been completed before the reorientation of the magnetization actually starts. Therefore, it is highly surprising that the route of the phase transition depends on the helicity of light. This phenomenon can hardly be explained in terms of inertia-driven spin orientation between two coexisting states as observed in $\mathrm{HoFeO}_{3}$ [19]. In the present experiment, the final state in which the system eventually ends up does not yet exist during the action of the laser pulse. Another mechanism of all-optical magnetization reversal, observed in metallic GdFeCo alloys [16], is based on heating the free electrons in the metal well above the Curie temperature and also cannot be effective in these dielectric orthoferrite samples.

In order to unveil the mechanism of the polarizationcontrollable phase transition in $(\mathrm{SmPr}) \mathrm{FeO}_{3}$, we examined in detail how the magnetization in the laser-created domain depends on the laser pulse fluence and the initial sample temperature. Figure 3(a) shows images of magnetic domains obtained at different temperatures of the sample $3.7 \mathrm{~ns}$ after the excitation with right- and left-handed circularly polarized pump pulses of energy $4.3 \mu \mathrm{J}$. One can see that a left-handed circularly polarized laser pulse forms a predominantly white domain if the sample temperature is below $20 \mathrm{~K}$ and a black domain if the sample temperature is above $20 \mathrm{~K}$. Similarly, an excitation with a right-handed circularly polarized pulse results in a black (a) $\mathrm{t}=3.7 \mathrm{~ns}, \mathrm{E}=4.3 \mu \mathrm{J}$

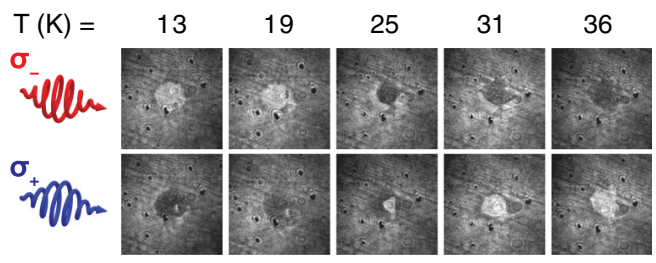

(b) $\mathrm{t}=3.7 \mathrm{~ns}, \mathrm{~T}=19 \mathrm{~K}$

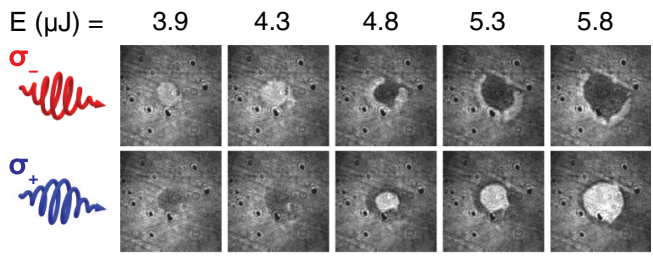

FIG. 3 (color online). Dependence of the laser-induced spin reorientation on temperature (a) and pump pulse energy (b) using pump pulses with right-handed $\left(\sigma_{+}\right)$and left-handed $\left(\sigma_{-}\right)$ circular polarization. The magneto-optical images show that, depending on the temperature $T$ and the pump pulse energy $E$, pulses of the same helicity can create domains with opposite magnetization.

domain below $20 \mathrm{~K}$ and in a white one above $20 \mathrm{~K}$. A similar result is obtained if one studies the laser-induced spin reorientation as a function of the pump fluence. Figure 3(b) shows that varying the pump fluence at a fixed temperature may lead to situations in which excitation by pump pulses of the same helicity results in the formation of magnetic domains with opposite orientations of $M_{z}$.

Based on these observations, we suggest that the creation of the magnetic domains proceeds via a yet unexplored mechanism. The control of the route of the phase transition is realized by the excitation of coherent, long-living spin precession, which dynamically breaks the equivalence between the routes towards the states with $M_{z}$ up and down. This is illustrated in Fig. 4. In the equilibrium state, at temperatures below $T_{1}$, the system is at the minimum of the thermodynamic potential with the magnetization aligned in the $x$ direction. A femtosecond circularly polarized pump pulse instantaneously excites spin precession via impulsive stimulated Raman scattering [20-22]. The amplitude of this precession is typically less than $10^{\circ}$, and the initial phase depends on the helicity of the pump polarization. At the same time, the absorbed light excites Fe $3 d$ electrons, which subsequently decay, generating a lot of nonequilibrium phonons within a few hundred femtoseconds. In the following time domain, two simultaneous processes are active in the material. First, the magnetization oscillates around the $x$ axis with a period of $\sim 10$ ps. Second, the nonequilibrium phonons interact with the RE ions, repopulating $4 f$ electronic sublevels of the ground state on a time scale given by the strength of the electronphonon interaction [18]. Thus, the effective temperature of 

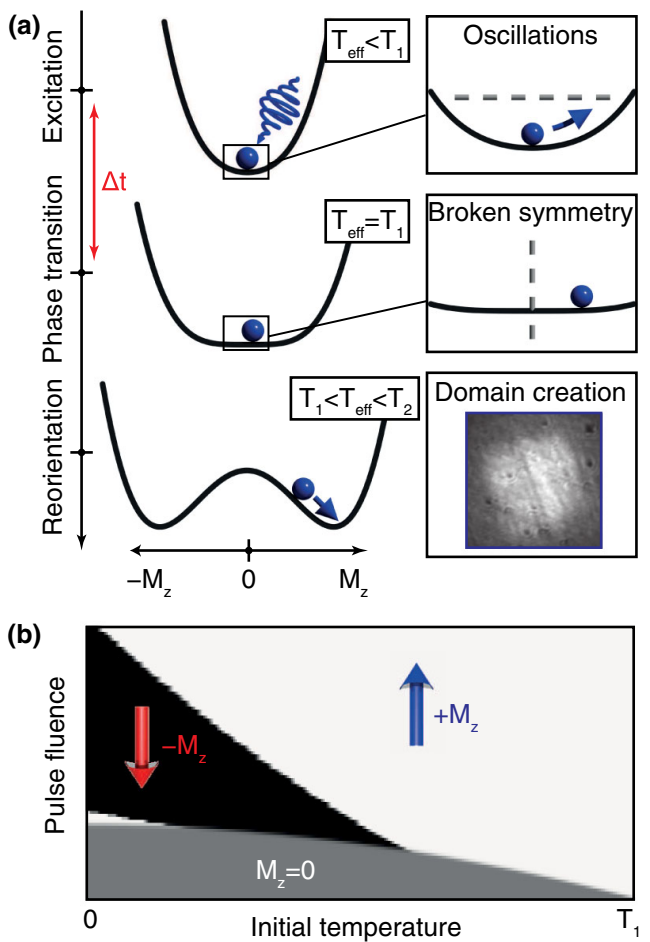

FIG. 4 (color online). (a) Illustration of the mechanism for controlling the path of a laser-induced phase transition. It is based on two effects from the same laser pulse: heating and the excitation of small amplitude spin precession. Because of heating, the effective temperature of the RE $4 f$ electrons $\left(T_{\text {eff }}\right)$ reaches the temperature of the first phase transition $\left(T_{1}\right)$ after time $\Delta t$. At that moment, the shape of the potential energy as a function of $M_{z}$ starts to change dramatically: The minimum of the potential, at $M_{z}=0$, splits into two minima, which separate further with increasing temperature. The spin precession is instantaneously excited at $t=0$. As the spins oscillate around their equilibrium, the symmetry is dynamically broken. Therefore, $M_{z}$ will be slightly closer to the left or the right minimum at the moment of the phase transition and will consequently proceed to grow in that direction. (b) The phase diagram, calculated by using a simple model of the mechanism, shows the reorientation direction as a function of sample temperature and pump pulse fluence after excitation with righthanded polarized light. The black and white colors represent a transition to a state in which $M_{z}$ is negative and positive, respectively. In the gray region, the laser-induced heating is insufficient to trigger the phase transition.

the RE $4 f$ electron system increases, and when it reaches $T_{1} \approx 98 \mathrm{~K}$ the initial equilibrium orientation of the magnetization along the $x$ axis becomes less favorable. Instead, two distinct minima arise, corresponding to states with opposite signs of $M_{z}$. As the heating of the RE ions progresses even further, the reorientation proceeds in the direction towards the nearest minimum. The coherent spin precession excited at $t=0$ breaks the symmetry in a controlled way: Whether $M_{z}$ is closer to the left or the right minimum is fully determined by the initial phase of the spin precession and the number of half periods that fit in the time $\Delta t$ between the excitation and the start of the reorientation.

Both the initial phase of the spin precession and $\Delta t$ are controlled by experimentally accessible parameters. To excite spin precession with the opposite initial phase, it is sufficient to use a laser pulse with the opposite helicity. This will always lead to a spin reorientation in the other direction. $\Delta t$ depends on the initial temperature and the laser pulse fluence, which determine, respectively, the required amount of heating and the speed of the heating. To illustrate how the reorientation direction can depend on these two parameters, we simulated this mechanism by using simple models for the speed of heating [18] and the temperature-dependent anisotropy [23]. We then solved the equations of motion of the excited system by using the Lagrangian discussed elsewhere [24,25] and calculated the magnetic state of the medium at $3 \mathrm{~ns}$ after excitation, as a function of laser pulse fluence and initial sample temperature. The results of the calculations are shown in Fig. 4(b). For high pump fluences or initial temperatures close to $T_{1}$, the reorientation proceeds in the direction that $M_{z}$ moves in directly after excitation. However, if the pump fluence is low and the heating is slow enough, the spin system has time for more than a half period of precession, which effectively leads to the opposite reorientation direction. A similar situation occurs when one changes the initial temperature at a fixed pump fluence, thus varying the time needed for the system to reach the point at which the spin reorientation begins. Therefore, it is the combination of helicity, temperature, and fluence that fully determines the final state of the excited system.

In conclusion, using a single-shot magneto-optical imaging technique, we have shown that the excitation of $(\mathrm{SmPr}) \mathrm{FeO}_{3}$ with a circularly polarized 60 fs laser pulse can effectively trigger an ultrafast spin reorientation and create a homogeneous magnetic domain with the orientation of the magnetization defined by the helicity of the laser pulse. This observation reveals the possibility to coherently control a magnetic phase transition and create magnetic domains without applying any magnetic field. We argue that the control is mediated by long-living coherent spin precession which is excited long before the phase transition takes place. A striking feature of this mechanism is that the route of the phase transition can be controlled through three parameters independently: the helicity of the laser pulse polarization, the initial temperature of the sample, or the laser pulse fluence. We would like to note that the mechanism demonstrated in this Letter may have implications for control of phase transitions in ferroelectric or multiferroic materials as well [26]. In contrast to magnetic phase transitions, the control of electric polarization can be mediated by coherent phonons, which can also be excited via impulsive stimulated Raman scattering [27]. 
We thank A. van Rooij, T. Toonen, and A. van Etteger for technical support. This work was partially supported by The Netherlands Organization for Scientific Research (NWO), the Foundation for Fundamental Research on Matter (FOM), the Russian Foundation for Basic Research (RFBR) Grant No. 10-02-01008-a, the European Union's Seventh Framework Program (FP7/2007-2013) Grants No. NMP3-SL-2008-214469 (UltraMagnetron), No. NMP3-LA-2010-246102 (IFOX), and No. 214810 (FANTOMAS) as well as the European Research Council under the European Union's Seventh Framework Program (FP7/2007-2013)/ERC Grant Agreement No. 257280 (Femtomagnetism). A.M.K. acknowledges the support from the Committee for Science and Education of the Government of St. Petersburg in 2010.

[1] C. M. Knobler and R. C. Desai, Annu. Rev. Phys. Chem. 43, 207 (1992).

[2] V.J. Anderson and H. N.W. Lekkerkerker, Nature (London) 416, 811 (2002).

[3] M. Wuttig and N. Yamada, Nature Mater. 6, 824 (2007).

[4] O. P. Uteza, E. G. Gamaly, A. V. Rode, M. Samoc, and B. Luther-Davies, Phys. Rev. B 70, 054108 (2004).

[5] N. Gedik, D.-S. Yang, G. Logvenov, I. Bozovic, and A. H. Zewail, Science 316, 425 (2007).

[6] P. Beaud, S. L. Johnson, E. Vorobeva, U. Staub, R. A. De Souza, C. J. Milne, Q.X. Jia, and G. Ingold, Phys. Rev. Lett. 103, 155702 (2009).

[7] A. V. Kimel, A. Kirilyuk, A. Tsvetkov, R. V. Pisarev, and T. Rasing, Nature (London) 429, 850 (2004).

[8] Y. Zhu, X. Zhang, T. Li, X. Huang, L. Han, and J. Zhao, Appl. Phys. Lett. 95, 052108 (2009).

[9] G. Ju, J. Hohlfeld, B. Bergman, R. J. M. van de Veerdonk, O. N. Mryasov, J.-Y. Kim, X. Wu, D. Weller, and B. Koopmans, Phys. Rev. Lett. 93, 197403 (2004).

[10] J.-U. Thiele, M. Buess, and C. H. Back, Appl. Phys. Lett. 85, 2857 (2004).
[11] A. M. Balbashov and S. K. Egorov, J. Cryst. Growth 52, 498 (1981).

[12] R. B. Clover, Jr., C. Wentworth, and S. S. Mroczkowski, IEEE Trans. Magn. 7, 480 (1971).

[13] R. B. Clover, Jr., "Low Birefringent Orthoferrites," U.S. Patent No. 3804766 (1974).

[14] I. Dzyaloshinsky, J. Phys. Chem. Solids 4, 241 (1958).

[15] T. Moriya, Phys. Rev. 120, 91 (1960).

[16] K. Vahaplar, A. M. Kalashnikova, A. V. Kimel, D. Hinzke, U. Nowak, R. Chantrell, A. Tsukamoto, A. Itoh, A. Kirilyuk, and T. Rasing, Phys. Rev. Lett. 103, 117201 (2009).

[17] P. A. Usachev, R. V. Pisarev, A. M. Balbashov, A. V. Kimel, A. Kirilyuk, and T. Rasing, Fiz. Tverd. Tela 47, 2200 (2005) [Phys. Solid State 47, 2292 (2005)].

[18] J. A. de Jong, A. V. Kimel, R. V. Pisarev, A. Kirilyuk, and T. Rasing, Phys. Rev. B 84, 104421 (2011).

[19] A. V. Kimel, B. A. Ivanov, R. V. Pisarev, P. A. Usachev, A. Kirilyuk, and T. Rasing, Nature Phys. 5, 727 (2009).

[20] A. V. Kimel, A. Kirilyuk, P. A. Usachev, R. V. Pisarev, A. M. Balbashov, and T. Rasing, Nature (London) 435, 655 (2005).

[21] A. M. Kalashnikova, A. V. Kimel, R. V. Pisarev, V. N. Gridnev, P. A. Usachev, A. Kirilyuk, and T. Rasing, Phys. Rev. B 78, 104301 (2008).

[22] R. Iida, T. Satoh, T. Shimura, K. Kuroda, B. A. Ivanov, Y. Tokunaga, and Y. Tokura, Phys. Rev. B 84, 064402 (2011).

[23] H. Horner and C. M. Varma, Phys. Rev. Lett. 20, 845 (1968).

[24] V. G. Baryakhtar, B. A. Ivanov, and M. V. Chetkin, Usp. Fiz. Nauk 146, 417 (1985) [Sov. Phys. Usp. 28, 563 (1985)].

[25] V. G. Baryakhtar, B. A. Ivanov, M. V. Chetkin, and S. N. Gadetskii, Dynamics of Topological Magnetic Solitons: Experiment and Theory (Springer-Verlag, Berlin, 1994).

[26] S.L. Johnson et al., Phys. Rev. Lett. 108, 037203 (2012).

[27] R. Merlin, Solid State Commun. 102, 207 (1997). 